\title{
Exponentially stable non-linear control for speed regulation of induction motor with field-oriented PI-controller
}

\author{
Hou-Tsan Lee ${ }^{1 *}$, Jaion-Shea Chang ${ }^{1}$ and Li-Chen $\mathrm{Fu}^{1,2}$ \\ ${ }^{1}$ Department of Electrical Engineering, National Taiwan University, Taipei, Taiwan, R.O.C. \\ ${ }^{2}$ Department of Computer Science and Information Engineering, National Taiwan University, Taipei, Taiwan, R.O.C.
}

\begin{abstract}
SUMMARY
On the basis of a novel dynamic model obtained under a special non-linear co-ordinate transform on a $d$-q-axis model (w.r.t. the stationary reference frame) of an induction motor, a simple but effective control law for speed regulation is proposed in this paper. This controller is designed using the concept of the field orientation and the property of linear PI-controller, and is shown to be robust with respect to variation of system parameters and load structure. To be rigorous, a Lyapunov theorem with a simple iterative criterion derived in this paper proves the local exponential stability of the system around any desired speed. Numerical simulations are also given to show the effectiveness of the presented control law. Copyright (C) 2000 John Wiley \& Sons, Ltd.
\end{abstract}

\section{INTRODUCTION}

Pioneered by Blaschke [1], field orientation is the most widely used method for servo control of induction motor, and field orientation techniques have been successfully used for induction motor control [2], just the same as the PID controller for linear systems. In most of the existing non-linear controllers for induction motors [3, 4], the rotor flux is made to converge asymptotically to a fixed constant, which is to prevent the saturation of the magnetic circuit. However, the flux saturation phenomenon remains due to the reluctant flux in the stator and the rotor [5], and hence the control schemes still can hardly achieve their original goal satisfactorily.

The non-linear adaptive control schemes are also applied when some imprecisely known parameters are concerned $[4,6]$, such as the rotor resistance, which changes with temperature variation, and the coefficients of the load characteristics. But since the control law will normally depend on the type of load structure, the form of the adaptive schemes will have to be changed when the load type is changed. Recently, the sensorless field oriented control scheme gradually became a popular control method for induction motors $[7,8]$. Some of them take the rotor resistance adaptation into the control scheme [9], but the complicated structures of the observers

*Correspondence to: Hou-Tsan Lee, Department of Electrical Engineering, National Taiwan University, Taipei, Taiwan, R.O.C.

Copyright (C) 2000 John Wiley \& Sons, Ltd. 
(or estimators) are often difficult to analyze and design. Taking the output (speed) feedback seemingly is another effective alternative in dealing with the problem of unknown resistance [10, 11]. Although the stator currents are needed, the speed and the flux can be tracked independently.

Given the above observation, here we try to devise a simple controller for the speed regulation of induction motor driving a more general class of load. The flux tracking is not considered here. With the robustness of general PI-control, the unknown parameters in the system, as mentioned previously, need not be estimated. Because the transformation between the actual input voltages and the $d-q$-axis voltages is stationary, it can be done easily by some appropriate analog circuit design. And, the computation time needed in the control process is relatively little. Therefore, the proposed control scheme has a higher potential to be successfully implemented with more ease. Finally, this paper analyses the stability property of this newly developed field-oriented linear PI-controller of induction motor, though its simpler version has already been widely employed in industry applications.

The paper is organized as follows. In Section 2, the $d$-q-axis electromechanical model of an induction motor is first introduced. Then, via a non-linear state transformation developed in this paper, a novel dynamic model is derived also in the same section, which is then integrated with a PI controller to yield a desirable equilibrium point. To analyse the stability type of the equilibrium point, some necessary mathematical preliminary is introduced in Section 3. In Section 4, the proof of the local exponential stability of the system around any desired speed is presented. The numerical simulations are given in Section 5 to demonstrate the effectiveness of the proposed controller. Finally, Section 6 contains some concluding remarks.

\section{FIELD-ORIENTED PI-CONTROLLER}

\subsection{Dynamical model of induction motor}

As is well known, the dynamical model of an induction motor can be simplified by a $d$ - $q$-axis co-ordinate transformation to some rotation-reference frame [5]. But for implentation feasibility, the stationary-reference frame is more popularly used. Thus, here we adopt the following group of $d-q$-axis coordinate-transformed dynamical equations of an induction motor [3]:

$$
\begin{aligned}
i_{q \mathrm{~s}} & =-\alpha_{1} i_{q \mathrm{~s}}+\alpha_{2} \lambda_{q \mathrm{r}}-\beta \omega_{\mathrm{r}} \lambda_{d \mathrm{r}}+c V_{q \mathrm{~s}} \\
\dot{i}_{d \mathrm{~s}} & =-\alpha_{1} i_{d \mathrm{~s}}+\beta \omega_{\mathrm{r}} \lambda_{q \mathrm{r}}+\alpha_{2} \lambda_{d \mathrm{r}}+c V_{d \mathrm{~s}} \\
\dot{\lambda}_{q \mathrm{r}} & =-\alpha_{3} i_{q \mathrm{~s}}-\alpha_{4} \lambda_{q \mathrm{r}}+\omega_{\mathrm{r}} \lambda_{d \mathrm{r}} \\
\dot{\lambda}_{d \mathrm{r}} & =-\alpha_{3} i_{d \mathrm{~s}}-\alpha_{4} \lambda_{d \mathrm{r}}-\omega_{r} \lambda_{q \mathrm{r}} \\
J_{\mathrm{m}} \dot{\omega}_{\mathrm{r}} & =T_{\mathrm{e}}-T_{\mathrm{L}} \\
T_{\mathrm{e}} & =K_{t}\left(\lambda_{d \mathrm{r}} i_{q \mathrm{~s}}-\lambda_{q \mathrm{r}} i_{d \mathrm{~s}}\right)
\end{aligned}
$$

where the states and the parameters are defined as shown in the nomenclature. 


\subsection{Analysis of mechanical load}

For general mechanical systems, the load torque is a strictly increasing function of the rotor speed $\omega_{\mathrm{r}}$ if the inertial part does not exist or were to be neglected. For example, the load torque of the fan-type load system can be modelled as ' $b_{0}+b_{1} \omega_{\mathrm{r}}+b_{2} \operatorname{sign}\left(\omega_{\mathrm{r}}\right) \omega_{\mathrm{r}}^{2}$ ' which is apparently a strictly increasing function of $\omega_{\mathrm{r}}$. For this reason, throughout the paper we will consider the class of mechanical loads, which satisfies the following assumption:

$$
T_{\mathrm{L}}=J_{\mathrm{L}} \dot{\omega}_{\mathrm{r}}+f_{\mathrm{L}}\left(\omega_{\mathrm{r}}\right) \quad \text { with } \quad \frac{\mathrm{d} f_{\mathrm{L}}\left(\omega_{\mathrm{r}}\right)}{\mathrm{d} \omega_{\mathrm{r}}}>0, \quad \forall \omega_{\mathrm{r}} \in R, \quad \text { and } \quad f_{\mathrm{L}}(0)=0
$$

where $J_{\mathrm{L}}$ is the mechanical inertia of the payload.

\subsection{Field-oriented control}

The basic concept of a field-oriented control of an induction motor at steady state is to find the input voltages $V_{d \mathrm{~s}}, V_{q \mathrm{~s}}$ such that $\left[\lambda_{q \mathrm{r}} \lambda_{d \mathrm{r}}\right]\left[V_{d \mathrm{~s}}, V_{q \mathrm{~s}}\right]^{\mathrm{T}}$ is maximum, in order to maximize the energy transfer (see Appendix A), under the constraint that $V_{q \mathrm{~s}}^{2}+V_{d \mathrm{~s}}^{2}$ is a fixed constant, where $\lambda_{q \mathrm{r}}$ (or $\lambda_{d \mathrm{r}}$ ) in the $q$ - (or $d$-) axis rotor flux $[1,12]$. Applying such a control concept to the input voltage design of an induction motor, an intuitive realization of the field-oriented control law is as follows.

$$
c V_{q \mathrm{~s}}=\frac{\lambda_{d \mathrm{r}}}{\sqrt{\lambda_{q \mathrm{r}}^{2}+\lambda_{d \mathrm{r}}^{2}}} V \quad \text { and } \quad c V_{d \mathrm{~s}}=\frac{-\lambda_{q \mathrm{r}}}{\sqrt{\lambda_{q \mathrm{r}}^{2}+\lambda_{d \mathrm{r}}^{2}}} V
$$

which clearly satisfies the voltage constraint ' $\left(V_{q \mathrm{~s}}^{2}+V_{d \mathrm{~s}}^{2}\right)=(V / c)^{2}$ ' at any time instant. Of course, at present $V$ is no longer a constant. Instead, it offers degree of freedom (DOF) control to the system, but normally it will converge to a constant when the system approaches the steady state.

On the other hand, we would like to show that, given a desired speed $\omega_{\mathrm{d}}$, there exists a proper constant $V$ such that the steady state of the system exactly achieves the purpose of speed regulation, i.e. $\omega_{\mathrm{r}}=\omega_{\mathrm{d}}$. To this end, we further simplify the dynamics shown in (1) by introducing a non-linear state transformation as follows:

$$
\begin{aligned}
& x_{1}=h^{2}\left(i_{q \mathrm{~s}}^{2}+i_{d \mathrm{r}}^{2}\right)+\beta^{2} h^{2}\left(\lambda_{q \mathrm{r}}^{2}+\lambda_{d \mathrm{r}}^{2}\right)+2 \beta h^{2}\left(i_{q \mathrm{~s}} \lambda_{q \mathrm{r}}+i_{d \mathrm{~s}} \lambda_{d \mathrm{r}}\right) \\
& x_{2}=\lambda_{q \mathrm{r}}^{2}+\lambda_{d \mathrm{r}}^{2} \\
& x_{3}=\beta h\left(\lambda_{q \mathrm{r}}^{2}+\lambda_{d \mathrm{r}}^{2}\right)+\left(i_{q \mathrm{~s}} \lambda_{q \mathrm{r}}+i_{d \mathrm{~s}} \lambda_{d \mathrm{r}}\right) \\
& x_{4}=h\left(i_{q \mathrm{~s}} \lambda_{d \mathrm{r}}-i_{d \mathrm{~s}} \lambda_{q \mathrm{r}}\right) \\
& x_{5}=\omega_{\mathrm{r}}
\end{aligned}
$$


where $h=D / L_{\mathrm{r}}$, under which the dynamical equations shown in (1) can be transformed to the following novel dynamic model:

$$
\begin{aligned}
& \dot{x}_{1}=-2 a_{1} x_{1}+2 a_{2} x_{3}+\frac{2 x_{4}}{\sqrt{x_{2}}} \\
& \dot{x}_{2}=-2 a_{4} x_{2}+2 a_{3} x_{3} \\
& \dot{x}_{3}=a_{3} x_{1}+a_{2} x_{2}-\left(a_{1}+a_{4}\right) x_{3}+x_{5} x_{4} \\
& \dot{x}_{4}=-x_{5} x_{3}-\left(a_{1}+a_{4}\right) x_{4}+\sqrt{x_{2}} V \\
& \dot{x}_{5}=a_{5} x_{4}-f\left(x_{5}\right),
\end{aligned}
$$

where the parameters $a_{1}, a_{2}, a_{3}, a_{4}, a_{5}$ and the function $f\left(x_{5}\right)$ are defined in the nomenclature. Thus, it can be verified that system (2) has $\left[x_{1 \mathrm{~s}}, x_{2 \mathrm{~s}}, x_{3 \mathrm{~s}}, x_{4 \mathrm{~s}}, x_{5 \mathrm{~s}}\right]$ as its unique equilibrium point, where

$$
\begin{aligned}
& x_{1 \mathrm{~s}}=\left(\frac{m}{a_{3}}\right)^{2}\left(a_{4}^{2}+z^{2}\right) \\
& x_{2 \mathrm{~s}}=m^{2} \\
& x_{3 \mathrm{~s}}=\frac{a_{4}}{a_{3}} m^{2} \\
& x_{4 \mathrm{~s}}=\frac{z}{a_{3}} m^{2} \\
& x_{5 \mathrm{~s}}=\omega_{\mathrm{d}}
\end{aligned}
$$

with

$$
\begin{aligned}
d & =a_{1} a_{4}-a_{2} a_{3} \\
z^{2}+\omega_{\mathrm{d}} z & =d \\
m & =\sqrt{\frac{a_{3} f\left(\omega_{\mathrm{d}}\right)}{a_{5} z}}
\end{aligned}
$$

under the condition:

$$
V=V_{0}^{*}=\frac{m}{a_{3}}\left[a_{4} \omega_{\mathrm{d}}+\left(a_{1}+a_{4}\right) z\right]
$$




\subsection{PI-controller for speed regulation}

Design of controller based on the system structure often involves complicated algorithms which may possibly lead to high implementation cost. This fact thus motivates the need of designing a simple PI-controller that can be applicable to a vast class of system structures. Now, we consider the load, which is modelled as in assumption (A1) and let the amplitude of the input voltage $V$ be designed as

$$
\begin{aligned}
& \dot{x}_{6}=k_{i}\left(\omega_{\mathrm{d}}-x_{5}\right) \\
& V=x_{6}+k_{p}\left(\omega_{\mathrm{d}}-x_{5}\right)
\end{aligned}
$$

By combining systems (2) and (5), when the combined system arrives at its equilibrium point $x_{\mathrm{s}}$, we have the following equations:

$$
x_{6 \mathrm{~s}}=V \quad \text { and } \quad 0=-x_{5 \mathrm{~s}} x_{3 \mathrm{~s}}-\left(a_{1}+a_{4}\right) x_{4 \mathrm{~s}}+\sqrt{x_{2 \mathrm{~s}}} V
$$

Thus, we can readily arrive at a conclusion that $x_{\mathrm{s}}=\left[x_{1 \mathrm{~s}}, x_{2 \mathrm{~s}}, x_{3 \mathrm{~s}}, x_{4 \mathrm{~s}}, x_{5 \mathrm{~s}}, x_{6 \mathrm{~s}}\right]^{\mathrm{T}}$, where $x_{i \mathrm{~s}}{ }^{\prime}$, $i=1, \ldots, 5$, is as defined in (3), and

$$
x_{6 \mathrm{~s}}=\left(\frac{m}{a_{3}}\right)\left[a_{4} \omega_{\mathrm{d}}+\left(a_{1}+a_{4}\right) z\right]
$$

is actually a unique equilibrium point of such an augmented system provided.

(A2) $a_{1}<3 a_{4}$ which will be justified later in Section 4 . In order to analyse the stability of the property of this equilibrium point, we will require some mathematical preliminaries, which will be shown in the next section.

To validate assumption (A2), we can use a linear current feedback to reduce the stator resistance $R_{\mathrm{s}}$, and hence, to reduce the parameter $a_{1}$. The details about the reason why the stator resistance can be reduced by a linear current feedback can be found in Reference [4].

(A3) $0.8 L_{\mathrm{s}} L_{\mathrm{r}} \leqslant L_{m}^{2}$,

The equilibrium point $x_{\mathrm{s}}$ is exponentially stable under proper design of the gains $k_{i}$ and $k_{p}$.

\section{Remark}

The air gap between the stator and the rotor is generally made sufficiently small so as to increase the efficiency of energy conversion between the electrical part and the mechanical part, and to reduce the reluctance (mainly caused by the air gap) significantly, which, in turn, implies that the ratio $L_{\mathrm{m}}^{2} / L_{\mathrm{r}} L_{\mathrm{s}}$ will approach unity. As a consequence, the above assumption is quite reasonable. 


\section{MATHEMATICAL PRELIMINARY}

In this section, we will develop a simple criterion to check whether a matrix $A_{\mathrm{e}}$ is Hurtwitz or not, where the matrix $A_{\mathrm{e}}$ is expanded as a given Hurwitz matrix, two vectors, and a scalar under a strictly positive real constraint. This result will be frequently used throughout the paper.

\section{Proposition 1}

Given a matrix $A \in R^{n \times n}$, two vectors $b \in R^{n \times 1}, c \in R^{1 \times n}$, and a scalar $d \in R$, if the matrix $A$ is Hurwitz and $R_{\mathrm{e}}\left\{-d-c(\mathrm{j} \omega I-A)^{-1} b\right\}>0$ for all $\omega \in R$, then the extended matrix

$$
A_{\mathrm{e}}=\left[\begin{array}{ll}
A & b \\
c & d
\end{array}\right]
$$

is Hurwitz.

Proof. To find the eigenvalues of $A_{\mathrm{e}}$, we assume that $\left|s I-A_{\mathrm{e}}\right|=0$. Thus, we have the determinant as follows:

$$
\left|\left[\begin{array}{cc}
s I-A & b \\
c & s-d
\end{array}\right]\right|=\left|\left[\begin{array}{cc}
s I-A & b \\
0 & s-d-c(s I-A)^{-1} b
\end{array}\right]\right|=0
$$

which means that $(s I-A)\left(s-d-(s I-A)^{-1} b\right)=0$. Because $A$ is Hurwitz, the real parts of $A$ 's eigenvalues are negative as given, and the last eigenvalue is $\lambda_{n+1}=d+c(\mathrm{j} \omega I-A)^{-1} b$ with $s=\mathrm{j} \omega$. Given $R_{\mathrm{e}}\left\{-d-c(\mathrm{j} \omega I-A)^{-1} b\right\}>0, \lambda_{n+1}$ is with negative real part. Therefore, all the eigenvalues of $A_{\mathrm{e}}$ are with negative real aparts. Hence, the matrix $A_{\mathrm{e}}$ is Hurwitz [13].

\section{Proposition 2}

Given polynomials $P(s), Q_{1}(s)$, and $Q_{2}(s)$. If both

$$
\mathrm{R}_{\mathrm{e}}\left(\frac{Q_{1}(\mathrm{j} \omega)}{P(\mathrm{j} \omega)}\right) \text { and } \mathrm{R}_{\mathrm{e}}\left\{\frac{Q_{2}(\mathrm{j} \omega)}{P(\mathrm{j} \omega)}\right\}
$$

are larger than zero for all $\omega \in R$, then we have

$$
\mathrm{R}_{\mathrm{e}}\left\{\frac{P(\mathrm{j} \omega)}{Q_{1}(\mathrm{j} \omega)+c Q_{2}(\mathrm{j} \omega)}\right\}>0
$$

for all $\omega \in \mathrm{R}$ and any $c>0$. 


\section{STABILITY ANALYSIS}

We now refer to the closed-loop system (2) and (5), which is rewritten as

$$
\begin{aligned}
& \dot{x}_{1}=-2 a_{1} x_{1}+2 a_{2} x_{3}+\frac{2 x_{4}}{\sqrt{x_{2}}} \\
& \dot{x}_{2}=-2 a_{4} x_{2}+2 a_{3} x_{3} \\
& \dot{x}_{3}=a_{3} x_{1}+a_{2} x_{2}-\left(a_{1}+a_{4}\right) x_{3}+x_{5} x_{4} \\
& \dot{x}_{4}=-x_{5} x_{3}-\left(a_{1}+a_{4}\right) x_{4}+\sqrt{x_{2}} V \\
& \dot{x}_{5}=a_{5} x_{4}-f\left(x_{5}\right) \\
& \dot{x}_{6}=k_{i}\left(\omega_{\mathrm{d}}-x_{5}\right) \\
& V=x_{6}+k_{p}\left(\omega_{\mathrm{d}}-x_{5}\right)
\end{aligned}
$$

System (7) has the equilibrium point $x_{\mathrm{s}}$ as defined above, for any given $\omega_{\mathrm{d}} \in R$, the block diagram of the control system is shown in Figure 1. Now, we are going to prove that the equilibrium point $x_{\mathrm{s}}$ is unique and is exponentially stable. The following theorem will give a complete statement of the conditions under which the aforementioned properties hold.

\section{Theorem 1}

For arbitrary desired speed $\omega_{\mathrm{d}} \in R$, suppose that the assumptions (A1)-(A3) are satisfied. If the controller (5) satisfies $16 d / 5 a_{4}<k_{p}<\left(a_{1}+a_{4}\right) / 2$, then $x_{\mathrm{s}}$ is the unique exponentially stable equilibrium point of system (7).

Proof. Since $x_{2}=\lambda_{q \mathrm{r}}^{2}+\lambda_{d \mathrm{r}}^{2}$ and $0 \cdot 8 L_{\mathrm{s}} L_{\mathrm{r}} \leqslant L_{\mathrm{m}}^{2}$, we always have $d>0.2 a_{1} a_{4}$ and $m \geqslant 0$. Without loss of generality, we assume that $\omega_{\mathrm{d}} \geqslant 0$.

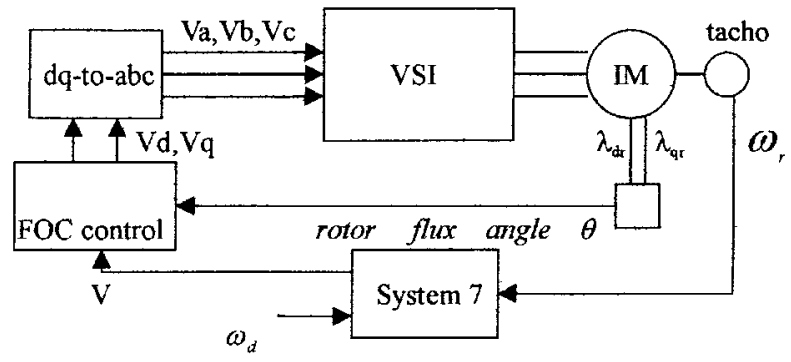

Figure 1. Block diagram of the control system. 
(1) Proof of uniqueness: For any given desired speed $\omega_{\mathrm{d}}, m=\sqrt{a_{3} f\left(\omega_{\mathrm{d}}\right) / a_{5} z}$ and the assumption on $f$, i.e. $f(0)=0$ and $\mathrm{d} f\left(\omega_{\mathrm{d}}\right) / \mathrm{d} \omega_{\mathrm{d}}>0$, imply that $\omega_{\mathrm{d}} z \geqslant 0$ and $z=\left(-\omega_{\mathrm{d}}+\sqrt{\omega_{\mathrm{d}}^{2}+4 d}\right) / 2$. Then, the equilibrium point $x_{\mathrm{s}}$ is unique if $x_{6 \mathrm{~s}}$ is a one-to-one function of $\omega_{\mathrm{d}}$. Define $g\left(\omega_{\mathrm{d}}\right)=\omega_{\mathrm{d}} / \sqrt{z}+\left(1+a_{1} / a_{4}\right) \sqrt{z}$, then we have the following relations:

$$
\begin{aligned}
x_{6 \mathrm{~s}} & =\left(\frac{a_{4}}{a_{3}}\right)\left(\sqrt{\frac{f\left(\omega_{\mathrm{d}}\right)}{a_{5}}}\left[\frac{\omega_{\mathrm{d}}}{\sqrt{z}}+\left(1+\frac{a_{1}}{a_{4}}\right) \sqrt{z}\right]\right. \\
& =\left(\frac{a_{4}}{a_{3}}\right)\left(\sqrt{\frac{f\left(\omega_{\mathrm{d}}\right)}{a_{5}}} g\left(\omega_{\mathrm{d}}\right)\right) \\
z^{\prime} & =\frac{\mathrm{d} z}{\mathrm{~d} \omega_{\mathrm{d}}}=\frac{-z}{\sqrt{\omega_{\mathrm{d}}+4 d}} \\
g^{\prime}\left(\omega_{\mathrm{d}}\right) & =\frac{\mathrm{d} g\left(\omega_{\mathrm{d}}\right)}{\mathrm{d} \omega_{\mathrm{d}}}=\frac{1}{\sqrt{z}}-\frac{\omega_{\mathrm{d}} z^{\prime}}{2 z^{3 / 2}}+\left(1+\frac{a_{1}}{a_{4}}\right)\left(\frac{z^{\prime}}{2 \sqrt{z}}\right) \\
& =\left(\frac{1}{2 \sqrt{z}}\right)\left[2+\frac{\omega_{\mathrm{d}}}{\sqrt{\omega_{\mathrm{d}}^{2}+4 d}}-\left(1+\frac{a_{1}}{a_{4}}\right) \frac{z}{\omega_{d}^{2}+4 d}\right]
\end{aligned}
$$

Since $a_{4}<3 a_{4}$ from (A2), we have

$$
g^{\prime}\left(\omega_{\mathrm{d}}\right) \geqslant \frac{1}{\sqrt{z}}\left(1-\frac{2 z}{\sqrt{\omega_{\mathrm{d}}^{2}+4 d}}\right)=\frac{\omega_{\mathrm{d}}}{z\left(\omega_{\mathrm{d}}^{2}+4 d\right)}>0
$$

Because $g^{\prime}\left(\omega_{\mathrm{d}}\right)>0$ for all $\omega_{\mathrm{d}}>0, g\left(\omega_{\mathrm{d}}\right)$ is a strictly increasing function of $\omega_{\mathrm{d}}$. Given the strictly increasing property of $g\left(\omega_{\mathrm{d}}\right)$ and $f\left(\omega_{\mathrm{d}}\right)$ with respect to $\omega_{\mathrm{d}}$, we can infer that $x_{6 \mathrm{~s}}$ is also a strictly increasing function of $\omega_{\mathrm{d}}$. As a result, $x_{6 \mathrm{~s}}$ is a one-to-one function of $\omega_{\mathrm{d}}$ and hence $x_{\mathrm{s}}$ is uniquely determined by $\omega_{\mathrm{d}}$.

(2) Proof of exponential stability: Let $J$ be the Jacobian matrix of the overall system at the equilibrium point $x_{\mathrm{s}}$ for an arbitrary desired speed $\omega_{\mathrm{d}}$, then we define $J^{\prime}=L J L^{-1}$ where

$$
L=\left[\begin{array}{cccc}
I_{4 \times 4} & \vdots & 0_{4 \times 2} & \\
\cdots & \vdots & \cdots & \cdots \\
0_{2 \times 2} & \vdots & 1 & 0 \\
& & -k_{p} & 1
\end{array}\right]
$$

Now, define $A_{n}$ to be the $n$th principal minor of the matrix $J^{\prime}$, then we want to prove that $J^{\prime}$ is Hurwitz through the following steps based on the results of Propositions 1 and 2.

Step 1: $A_{2}$ is Hurwitz for any given $\omega_{\mathrm{d}}$. This result can be checked easily.

Step 2: $A_{3}$ is Hurwitz for any given $\omega_{\mathrm{d}}$. 
Rewrite matrix $A_{3}$ as

$$
\left[\begin{array}{cc}
A_{2} & b_{2} \\
c_{2} & -\left(a_{1}+a_{4}\right)
\end{array}\right]
$$

then we have the following inequality by assumptions (A1)-(A3):

$$
\operatorname{Re}\left\{a_{1}+a_{4}-c_{2}\left(\mathrm{j} \omega I-A_{2}\right)^{-1} b_{2}\right\}>0, \quad \forall \omega \in R .
$$

By virtue of Proposition 1, we can conclude that matrix $A_{3}$ is Hurwitz for all $\omega_{\mathrm{d}} \in R$.

Step 3: By the same procedures, we can find that $J^{\prime}$ and $A_{n}$ are Hurwitz for $n=4,5$. By Lyapunov's direct theorem (application of converse theorems), the equilibrium point $x_{\mathrm{s}}$ is locally exponentially stable for any desired speed $\omega_{\mathrm{d}} \in R$. The proof is hence completed.

\section{COMPUTER SIMULATION}

(A) In this section, the performance of the presented controller, as it is applied to an induction motor, will be demonstrated by a number of simulation examples. The characteristics of the motor are listed below:

$R_{\mathrm{s}}=3.745, R_{\mathrm{r}}=3.745, L_{\mathrm{s}}=0.1633, L_{\mathrm{r}}=0.1633, L_{\mathrm{m}}=0.15467, P=6, J_{\mathrm{m}}=0.0284,6$ poles, $220 \mathrm{~V}$ AC.
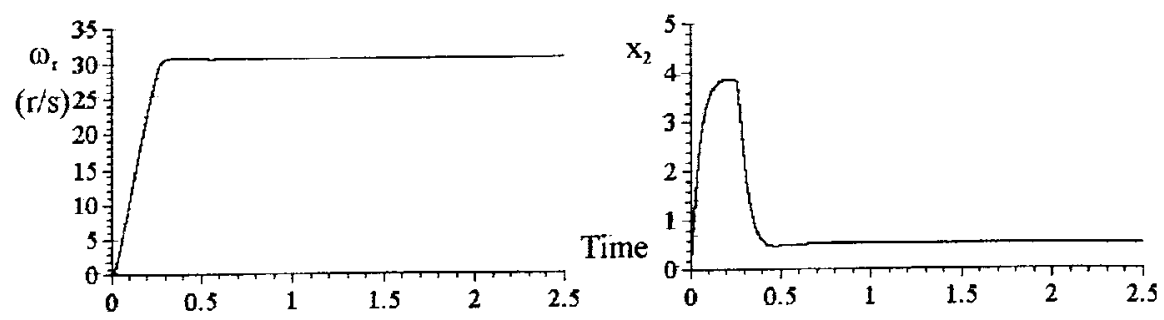

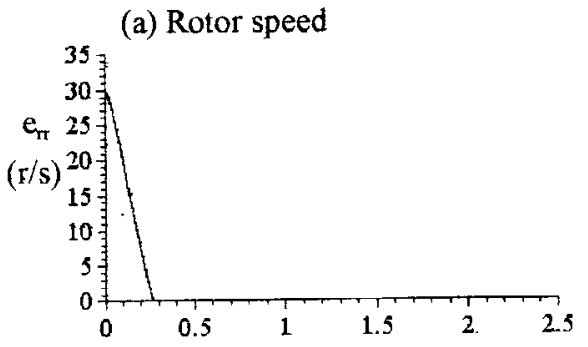

(c) Speed error

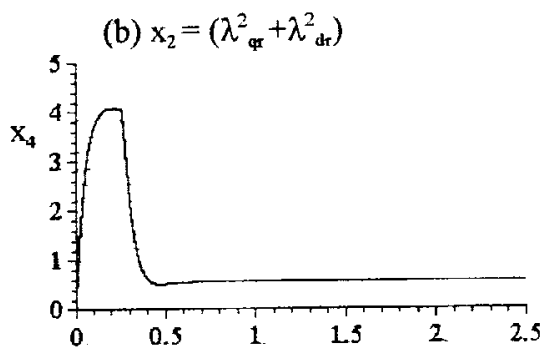

(d) $x_{4}$, proportionat to $T_{0}$

Figure 2. Responses and system (7) with $k_{i}=12.5$ and $k_{p}=3.4 d / a_{4}$ : (a) rotor speed; (b) $x_{2}=\left(\lambda_{\mathrm{qr}}^{2}+\lambda_{d \mathrm{r}}^{2}\right)$; (c) speed error; and (d) $x_{4}$, proportional to $T_{\mathrm{e}}$. 
The mechanical load torque is chosen to be $T_{\mathrm{L}}=J_{\mathrm{L}} \dot{\omega}_{\mathrm{r}}+b_{0}+b_{1} \omega_{\mathrm{r}}+b_{2} \operatorname{sign}\left(\omega_{\mathrm{r}}\right) \omega_{\mathrm{r}}^{2}$, whose, parameters are listed as follows: $J_{\mathrm{L}}=0.01, b_{0}=0, b_{1}=0.05, b_{2}=0.005$.

For practical implementation, the amplitude of the input voltage is limited. In our case, the upper bound of the input voltage is $300 \mathrm{~V}$. The desired speed $\omega_{\mathrm{d}}$ is $30\left(\mathrm{rad} \mathrm{s}^{-1}\right)$ (about $\left.286 \mathrm{rpm}\right)$ and the control gain $k_{p}$ is selected as $k_{p}=(3.4 \times d) / a_{4}$. Through a series of trial-and-error, we choose $k_{i}$ as designed to be 12.5 . It will minimize the overshoot of the system and have an acceptable response time, as shown in Figure 2(a). The rotor flux is a rather large number as shown in Figure 2(b), the same as the electric torque, which is proportional to $x_{4}\left(T_{\mathrm{e}}=\left(K_{t} / h\right) x_{4}\right)$, as shown in Figure 2(d). On the other hand, we propose a strategy to reduce the stator currents and rotor fluxes, which may cause the magnetic circuit to be saturated, and hence, greater power rating of the driver is required. This is similar to the so-called 'soft-start' problem in industrial applications, which can reduce the large initial speed error, which causes the large transient currents. We choose the new speed command, as shown in Figure 3(c), to ensure the speed error with $2\left(\mathrm{rad} \mathrm{s}^{-1}\right)$. After these arrangements, we have the simulation result as shown in Figure 3. The rotor flux and electric torque are reduced significantly as shown in Figures 3(b) and 3(d), but at the expense of large rise-time, as shown in Figure 3(a).

To show the robustness of the controller with respect to variation of system and load structure, we take the simulation cases as follows:

(1) Variation of $R_{\mathrm{r}}^{\prime}$ : The rotor resistance $R_{\mathrm{r}}$ suddenly increases to $1 \cdot 5 R_{\mathrm{r}}$ in the time interval [1 1.5$]$. Figure 4(a) shows that the controller is robust to the variation of rotor resistance $R_{\mathrm{r}}$. Only the electric torque slightly changes with it, as shown in Figure 4(b).

(2) Disturbance injection: The disturbance $T_{\mathrm{d}}=0.2(\mathrm{~N} \mathrm{~m})$ occurs at the time interval [ $\left[\begin{array}{ll}1 & 2\end{array}\right]$. As shown in Figure 5(a), the speed is recovered quickly as it never happened. The cost of maintaining the speed is the increase of electric torque, which is shown in Figure 5(b).

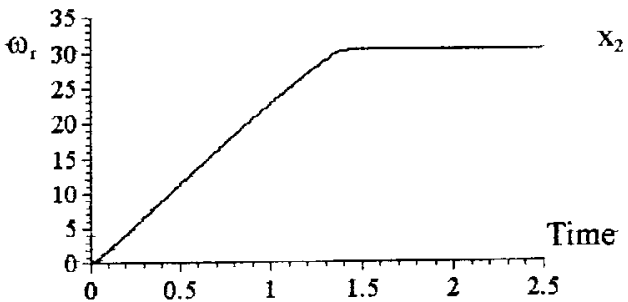

(a) Rotor speed

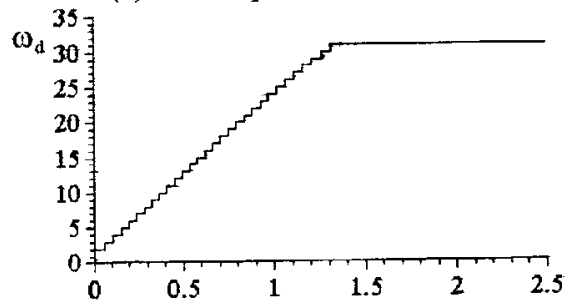

(c) Speed command

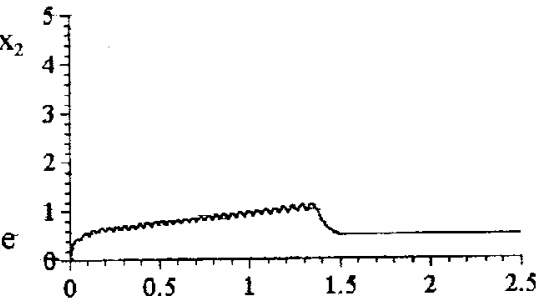

(b) $\mathrm{x}_{2}=\left(\lambda_{\mathrm{qr}}^{2}+\lambda_{\mathrm{dr}}^{2}\right)$

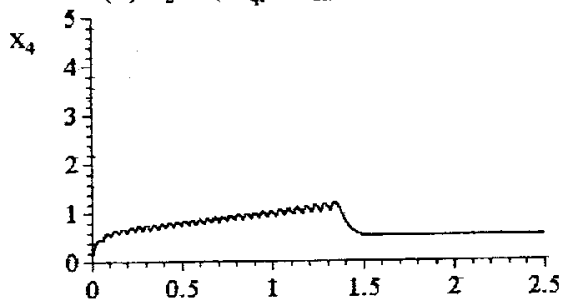

(d) $\mathrm{x}_{4}$, proportional to $\mathrm{T}_{\mathrm{e}}$

Figure 3. Responses of system (7) with new speed command and same $k_{t}, k_{p}$ as in Figure 2: (a) rotor speed; (b) $x_{2}=\left(\lambda_{q \mathrm{r}}^{2}+\lambda_{d \mathrm{r}}^{2}\right)$; (c) speed command; and (d) $x_{4}$, proportional to $T_{\mathrm{e}}$. 

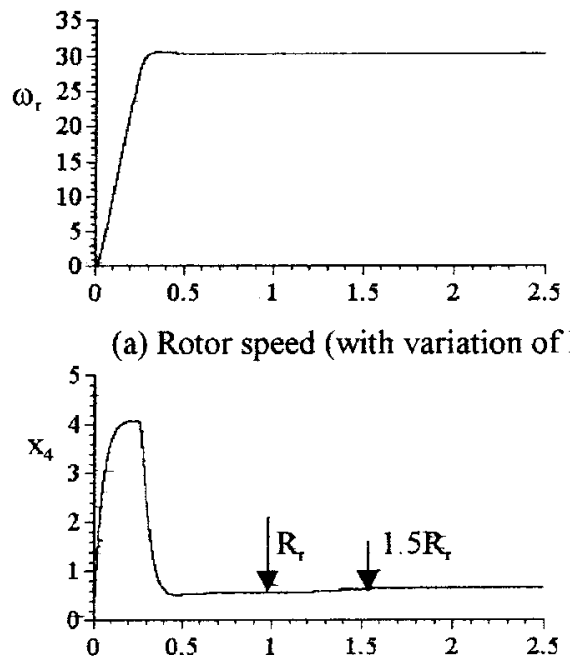

(b) $\mathrm{x}_{4}$, proportional with $\mathrm{T}_{\mathrm{e}}$

Figure 4. Responses of system (7) with variation of $R_{\mathrm{r}}$ (at time interval [ [ $\left.\begin{array}{lll}1 & 1 & 1.5\end{array}\right]$ ): (a) rotor speed (with variation of $R_{\mathrm{r}}$ ), and (b) $x_{4}$, proportional to $T_{\mathrm{e}}$.

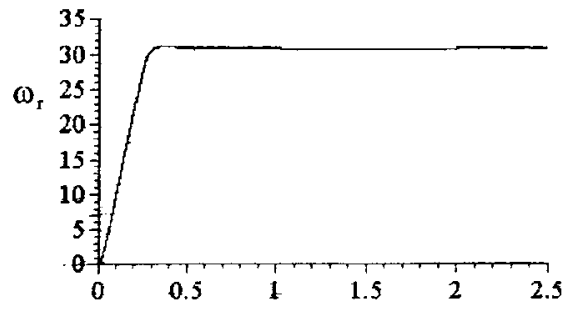

(a) Rotor speed (with disturbance)

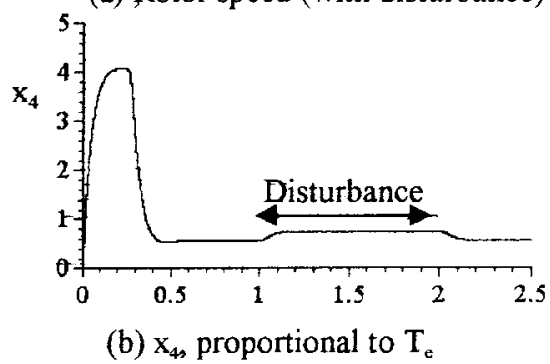

Figure 5. Responses of system (7) with $T_{\mathrm{d}}=0.1 \mathrm{~N} \mathrm{~m}$ at time interval [1 2 2]: (a) rotor speed (with disturbance), and (b) $x_{4}$, proportional to $T_{\mathrm{e}}$.

(B) Secondary, this controller is also applied to the benchmark experiment offered by IEEE Trans. On Industrial Electronics. The parameters of this motor are as follows: $R_{\mathrm{s}}=8, \mathbf{R}_{\mathrm{r}}=3.6$, $L_{\mathrm{s}}=0.47, L_{\mathrm{r}}=0.47, L_{\mathrm{m}}=0.44, \mathrm{np}=2, J_{\mathrm{m}}=0.06$, mechanic viscous damping constant $=0.04$, and the leakage factor $\sigma=0.12$. The nominal value for this machine are: 
rotor shaft velocity $\omega_{\text {norm }}=700 \mathrm{rev} \mathrm{min}^{-1} \quad\left(73.3038 \mathrm{rad} \mathrm{s}^{-1}\right)$,

torque $\tau_{\text {norm }}=7 \mathrm{~N} \mathrm{~m}, \quad$ stator current $\left\|i_{\mathrm{s}}\right\|=4.6 \mathrm{~A}$

stator voltage $\|u\|=180 \mathrm{~V}$, rotor flux norm $\beta_{\text {norm }}=1.22 \mathrm{~Wb}$

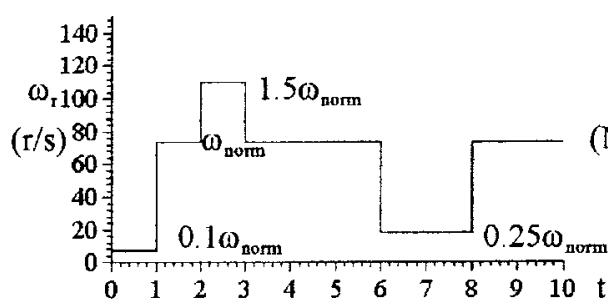

(a) Rotor speed command

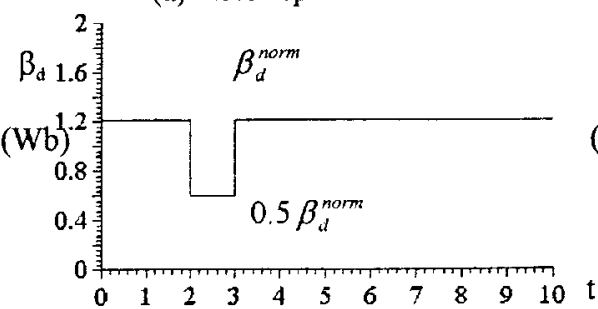

(c) Flux norm

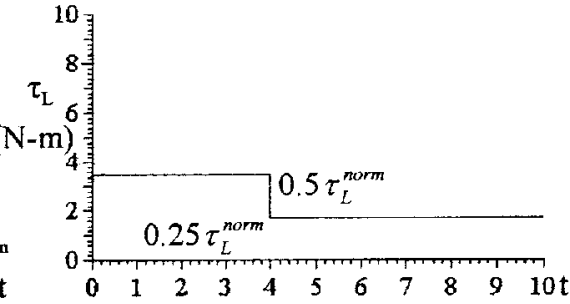

(b) Load torque variation

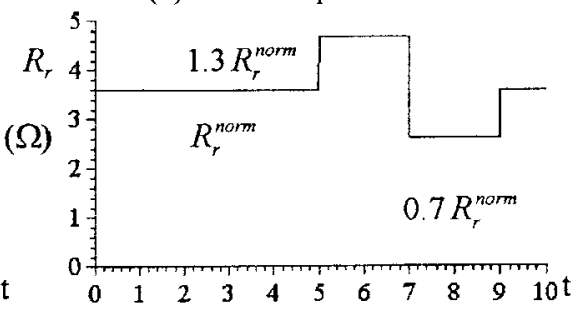

(d) Variation of $R_{r}$

Figure 6. Conditions of benchmark experiment: (a) rotor speed command, (b) load torque variation, (c) flux norm and (d) variation of $R_{\mathrm{r}}$.

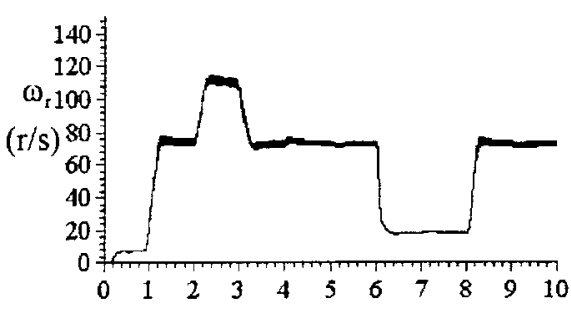

(a) Rotor speed

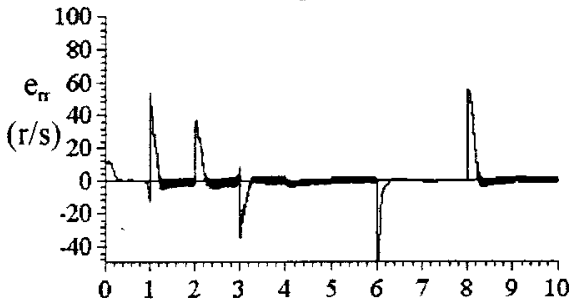

(c) Rotor speed error

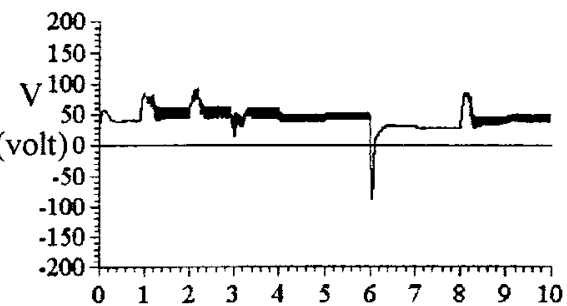

(b) Voltage input

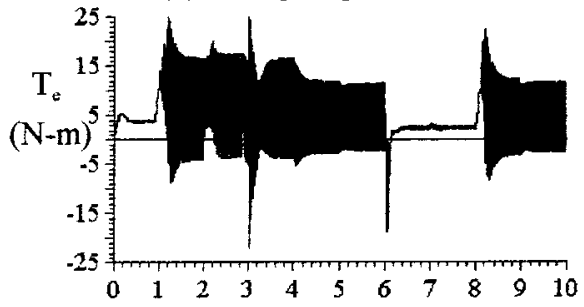

(d) Electrical torque

Figure 7. Simulation results of system (7) with benchmark experiment of Figure 6: (a) rotor speed, (b) voltage input, (c) rotor speed error, and (d) electrical torque. 


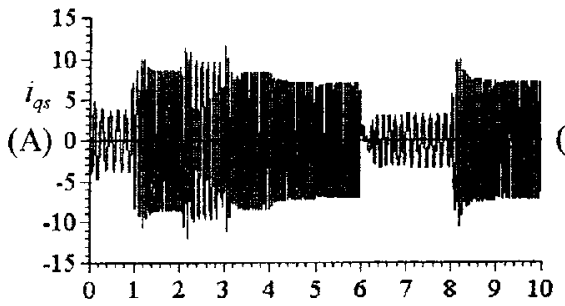

(a) Stator current $i_{q s}$

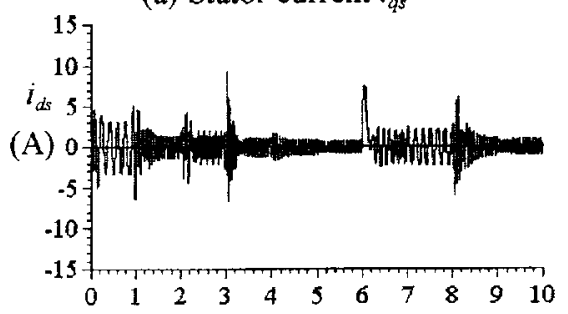

(c) Stator current $i_{d s}$

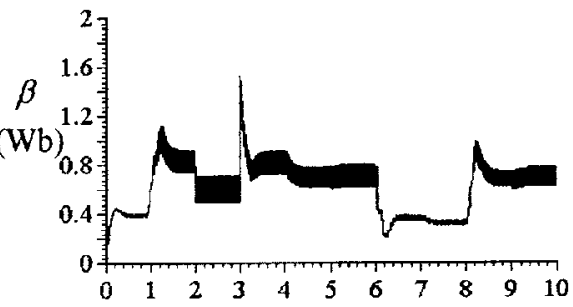

(b) Flux norm

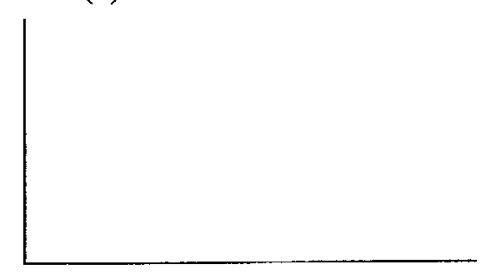

Figure 8. Simulation results of system with benchmark experiment of Figure 6 (continuous): (a) stator current $i_{q \mathrm{~s}}$ (b) flux norm, and (c) stator current $i_{d s}$.

Maximal values:

torque $|\tau| \leqslant 25 \mathrm{~N} \mathrm{~m}, \quad$ rotor flux norm $\beta_{\max }=\beta_{\text {norm }}=1.22 \mathrm{~Wb}$

Stator current $\left\|i_{\mathrm{s}}\right\| \leqslant 12 \mathrm{~A}, \quad$ stator voltage $\|u\| \leqslant 300 \mathrm{~V}$

Because the flux tracking is not involved in our theoretical analysis, we take $\beta_{\mathrm{d}}$ as a constraint $\left(\beta=\sqrt{\lambda_{d \mathrm{r}}^{2}+\lambda_{q \mathrm{r}}^{2}}\right)$. Under such conditions, we track the rotor speed command, as shown in Figure 6. Using the techniques developed in this paper, choose $k_{i}=100$ (if low speed under $0.1 \omega_{\text {norm }}$, $\left.k_{i}=500\right)$ and $k_{p}=(3.4 \times d) / a_{4}$. We have the simulation results, as shown in Figures 7 and 8 .

\section{CONCLUSION}

In this paper, we have presented a novel field-oriented PI-controller for an induction motor, which can drive a wide class of loads with good disturbance rejection property. Though it is difficult to prove the global stability of the overall system through Lyapunov analysis, we have proposed a simple iterative algorithm to prove that the system has a locally exponentially stable equilibrium point at any desired speed. Moreover, the control law is independent of the rotor resistance (a parameter, which normally varies with the temperature) and only uses the rotor speed and the orientation of rotor flux as feedback information, which hence becomes a plausible scheme. In additional, the benchmark experiment is also taken into a simulation. The further work is to deal with the torque and flux norm (as state $x_{4}, x_{2}$ ) regulation [14]. 


\section{APPENDIX A}

Let the dynamic equations of induction motor be with $d-q$ frame, which is rotating in $\omega_{\mathrm{s}}$. And, suppose $\bar{i}_{\mathrm{s}}$ only exist in $d$-axis, we have the spatial vector diagram as shown in Figure A1.

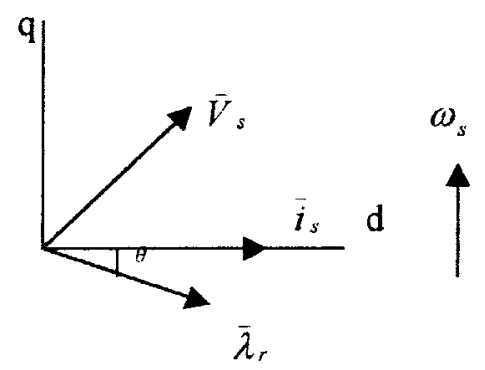

Figure A1. Spatial vector diagram of induction motor.

The dynamic equation of the stator voltage is expressed as follows:

$$
\bar{V}_{\mathrm{s}}=R_{\mathrm{s}} \bar{i}_{\mathrm{s}}+\frac{\mathrm{d} \bar{\lambda}_{\mathrm{s}}}{\mathrm{d} t}+\mathrm{j} \omega \bar{\lambda}_{\mathrm{s}}
$$

where the relationship between the flux and the currents is shown below:

or

$$
\bar{\lambda}_{\mathrm{s}}=L_{\mathrm{s}} \bar{i}_{\mathrm{s}}+L_{\mathrm{m}} \bar{i}_{\mathrm{r}}, \quad \text { and } \quad \bar{\lambda}_{\mathrm{r}}=L_{\mathrm{m}} \bar{i}_{\mathrm{s}}+L_{\mathrm{r}} \bar{i}_{\mathrm{r}}
$$

$$
\bar{\lambda}_{\mathrm{s}}=L_{\sigma} \bar{i}_{\mathrm{s}}+\frac{L_{\mathrm{m}}}{L_{\mathrm{r}}} \bar{i}_{\mathrm{r}}
$$

with

$$
L_{\sigma}=L_{\mathrm{s}}-\frac{L_{\mathrm{m}}^{2}}{L_{\mathrm{r}}}
$$

Substituting relationship (A2) into (A1), we have the new equation given below:

$$
\bar{V}_{\mathrm{s}}=R_{\mathrm{s}} \bar{i}_{\mathrm{s}}+\frac{\mathrm{d}}{\mathrm{d} t}\left(\frac{L_{\mathrm{m}}}{L_{\mathrm{r}}} \bar{\lambda}_{\mathrm{r}}+L_{\sigma} \bar{i}_{\mathrm{s}}\right)+\mathrm{j} \omega_{\mathrm{s}}\left(\frac{L_{\mathrm{m}}}{L_{\mathrm{r}}} \lambda_{\mathrm{r}}+L_{\sigma} \bar{i}_{\mathrm{s}}\right)
$$

Since the stator current only exists in the $d$-axis, then $\bar{i}_{\mathrm{s}}$ can be viewed as a scalar $i_{\mathrm{s}}$, which along with the vector $\bar{\lambda}_{\mathrm{r}}=\lambda_{\mathrm{r}} \mathrm{e}^{-\mathrm{j} \theta}$ can $\mathrm{b}$ substituted into the above equation. After separating the real part from the imaginary part in the equation, we end up with the two equations given below:

$$
\begin{aligned}
& V_{\mathrm{ds}}=R_{\mathrm{s}} i_{\mathrm{s}}+L_{\sigma} \frac{\mathrm{d}}{\mathrm{d} t} i_{\mathrm{s}}+\frac{L_{\mathrm{m}}}{L_{\mathrm{r}}} \lambda_{\mathrm{r}}\left(\omega_{\mathrm{s}}-\frac{\mathrm{d} \theta}{\mathrm{d} t}\right) \sin \theta \\
& V_{q \mathrm{~s}}=\omega_{\mathrm{s}} L_{\sigma} i_{\mathrm{s}}+\frac{L_{\mathrm{m}}}{L_{\mathrm{r}}} \lambda_{\mathrm{r}}\left(\omega_{\mathrm{s}}-\frac{\mathrm{d} \theta}{\mathrm{d} t}\right) \cos \theta
\end{aligned}
$$


Rearranging equation (A3) with $\lambda_{d \mathrm{r}}=\lambda_{\mathrm{r}} \cos \theta$ and $\lambda_{q \mathrm{r}}=\lambda_{\mathrm{r}} \sin \theta$, we achieve the new equation(A4) as given below:

$$
\begin{aligned}
& \lambda_{q \mathrm{r}}=\left[\frac{L_{\mathrm{m}}}{L_{\mathrm{r}}}\left(\omega_{\mathrm{s}}-\frac{\mathrm{d} \theta}{\mathrm{d} t}\right)\right]^{-1}\left(V_{d \mathrm{~s}}-R_{\mathrm{s}} i_{\mathrm{s}}-L_{\sigma} \frac{\mathrm{d} i_{\mathrm{s}}}{\mathrm{d} t}\right) \\
& \lambda_{d \mathrm{r}}=\left[\frac{L_{\mathrm{m}}}{L_{\mathrm{r}}}\left(\omega_{\mathrm{s}}-\frac{\mathrm{d} \theta}{\mathrm{d} t}\right)\right]^{-1}\left(V_{q \mathrm{~s}}-\omega_{\mathrm{s}} L_{\sigma}-i_{\mathrm{s}}\right)
\end{aligned}
$$

Choose $\left.k=\left(L_{\mathrm{m}} / L_{\mathrm{r}}\right)\left(\omega_{\mathrm{s}}-\mathrm{d} \theta / \mathrm{d} t\right)\right]^{-1}$, then $k$ is positive in steady state since $\omega_{\mathrm{s}}>\mathrm{d} \theta / \mathrm{d} t$ for all sufficiently long $t$. To maximize the matrix product

$$
\left[\begin{array}{ll}
\lambda_{q \mathrm{r}} & \lambda_{d \mathrm{r}}
\end{array}\right]\left[\begin{array}{ll}
V_{d \mathrm{~s}} & V_{q \mathrm{~s}}
\end{array}\right]^{\mathrm{T}}
$$

it is equivalent to the following problem:

$$
\begin{aligned}
& \max \left\{k\left(V_{d \mathrm{~s}}^{2}-R_{\mathrm{s}} i_{\mathrm{s}} V_{d \mathrm{~s}}-V_{d \mathrm{~s}} L_{\sigma} \frac{\mathrm{d} i_{\mathrm{s}}}{\mathrm{d} t}+V_{q \mathrm{~s}}^{2}-L_{\sigma} V_{q \mathrm{~s}} i_{\mathrm{s}}\right)\right\} \\
& =\max \left\{k\left(V^{2}-\mathrm{Loss}\right)\right\}
\end{aligned}
$$

Therefore, the energy transfer is maximal when $\left[\begin{array}{ll}\lambda_{q \mathrm{r}} & \lambda_{d \mathrm{r}}\end{array}\right]\left[\begin{array}{ll}V_{d \mathrm{~s}} & V_{q \mathrm{~s}}\end{array}\right]^{\mathrm{T}}$ is maximal.

\section{APPENDIX B: NOMENCLATURE}

$$
\begin{array}{ll}
i_{q \mathrm{~s}}\left(i_{d \mathrm{~s}}\right) & q-(d-) \text { axis input stator current } \\
V_{q \mathrm{~s}}\left(V_{d \mathrm{~s}}\right) & q-(d-) \text { axis input stator voltage } \\
\lambda_{q \mathrm{r}}\left(\lambda_{d \mathrm{r}}\right) & q \text {-(d-) axis rotor flux } \\
T_{\mathrm{e}} & \text { electromagnetic torque } \\
L_{\mathrm{m}} & \text { mutual inductance } \\
J_{\mathrm{m}} & \text { inertia of the motor } \\
D=\left(L_{\mathrm{s}} L_{\mathrm{r}}-L_{\mathrm{m}}^{2}\right) & \\
R_{\mathrm{s}}\left(R_{\mathrm{r}}\right) & \text { stator (rotor) resistance } \\
L_{\mathrm{s}}\left(L_{\mathrm{r}}\right) & \text { stator (rotor) inductance } \\
\omega_{\mathrm{r}} & \text { rotor speed } \\
T_{\mathrm{L}} & \text { mechanical load torque } \\
P & \text { number of poles } \\
J_{\mathrm{L}} & \text { inertia of the load } \\
\rho=\left(1-L_{\mathrm{m}}^{2} / L_{\mathrm{s}} L_{\mathrm{r}}\right) & \\
\beta=L_{\mathrm{m}} / D, & \\
\alpha_{1}=\left(R_{\mathrm{s}} L_{\mathrm{r}}\right) / D+\beta \alpha_{3}, & \\
\alpha_{3}=L_{\mathrm{m}} \alpha_{4}, & \\
K_{t}=3 P L_{\mathrm{m}} / 4 L_{\mathrm{r}}, & \\
a_{2}=L_{\mathrm{m}} R_{\mathrm{s}} / D, &
\end{array}
$$




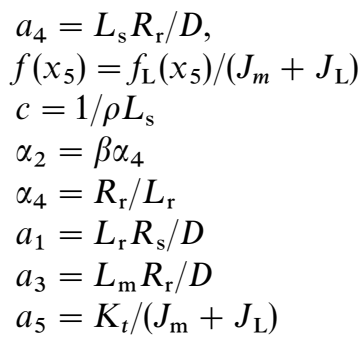

\section{REFERENCES}

1. Blaschke F. The principle of field orientation applying to the new transvector closed-loop control system for rotation field machines. Siemens-Review 1972; 39: 217-220.

2. Leonard W. Control of Electrical Drives. Springer: New York, 1985.

3. Kanellakopoulos I, Krein PK. Integral-action nonlinear control of induction motors. 12th IF AC World Congress, Sydney, 1993; 251-254.

4. Ortega R, Canudas C, Seleme S. Nonlinear control of induction motors: torque tracking with unknown load disturbance. American Control Conference, Chicago, IL, 1992; 206-210.

5. Krause PC. Analysis of Electric Machinery. McGraw-Hill: New York, 1986.

6. Mario RM, Peresada S, Valigi P. Adaptive input-output linearlization control of induction motors. IEEE Transactions in Automatic Control 1992; 38(2): 208-221.

7. Tajima H, Hori Y. Speed sensorless field-orientation control of induction machine. IEEE Transactions on Industrial Applications 1993; 29(1): 175-180.

8. Bodson M, Chiasson J, Novotnak RT. Nonlinear speed observer for high-performance induction motor control. IEEE Transactions on Industrial Electronics 1995; 42(4): 337-343.

9. Kubota H, Matsuse K. Speed sensorless field-oriented control of induction motor with rotor resistance adaptation. IEEE Transactions on Industrial Applications 1994; 30(5): 1219-1224.

10. Mario RM, Peresada S, Tomei P. Adaptive observer-based control of induction motor with unknown rotor resistance. International Journal of Adaptive Control and Signal Processing 1996; 10: 345-363.

11. Mario RM, Peresada S, Valigi P. Output feedback control of current-fed induction motors with unknown rotor resistance. IEEE Transactions on Control Systems Technology 1996; 4(4): 336-347.

12. Bassi E, Benzi FP, Bolobnani S, Buja GS. A field orientation scheme for current-fed induction motor drives based on the torque angle closed-loop control. IEEE Transactions on Industrial Applications 1992; 28(5): 1038-1044.

13. Vidyasagar M. Nonlinear Systems Analysis. Prentice-Hall: New Jersey, 1993.

14. Espinosa G, Ortega R, Nicklasson PJ. Torque and flux tracking of induction motors. International Journal of Robust and Nonlinear Control 1997; 7: 1-9.

15. Boyd S, Yang Q. Structure and simultaneous Lyapunov function for system stability problems. International Journal of Control 1989; 49(6): 2215-2240. 